\title{
Heterogeneous Responses to China and Oil Shocks: the G7 Stock Markets
}

\author{
Jamal Bouoiyour \\ IRMAPE, ESC Pau Business School, France \\ CATT, University of Pau, Pau, France \\ Refk Selmi \\ IRMAPE, ESC Pau Business School, France \\ CATT, University of Pau, Pau, France
}

\begin{abstract}
Given its size and integration with the global economy, Chinese economic downturn could have momentous spillovers to the rest of the world and result in a decline in oil prices. This article investigates whether the Chinese economic slowdown and the oil prices affect the G7 stock market. We use a Quantile-on-Quantile regression approach to capture the correlation structure between the G7 stock returns and oil price returns under different G7 market conditions with considering nuances of oil price movements and Chinese slowdown. Data are employed over the period of January $1999 \sim$ December 2015. Our results show that the responses of G7 stock returns to China and oil shocks are likely to be asymmetric, nonlinear and country-specific. The stock market returns of Germany, Italy and Canada appear the most vulnerable to these shocks. Our results suggest that international investors consider the states of stock market returns and oil price alongside with the interaction effect between China's economic slowdown and oil market.
\end{abstract}

\footnotetext{
* CorrespondingAuthor: Jamal Bouoiyour; Avenue du Doyen Poplawski, 64000 Pau, France, Tel: +33 0559408001, Fax: +33 0559408010, E-mail: jamal.bouoiyour@univ-pau.fr

Co-Author : Refk Selmi ; Avenue du Doyen Poplawski, 64000 Pau, France, Tel: +33 0559408001, Fax: +33 0559408010, E-mail: refk selmi@univ-pau.fr.
}

Acknowledgement: The authors would like to thank the editor-in chief and the anonymous reviewer for providing us in-depth comments, which have improved the quality of the paper. 
JEL Classification: F30, F36, G11, G15.

Keywords: G7 stock markets, Chinese economic slowdown, Oil shocks, Quantile-on-Quantile regression.

\section{Introduction}

Higher volatility caused by geostrategic concerns such as terrorist attack and economic downturn such as Chinese worsening outlook in these days evokes fear that makes it difficult to allocate investment optimally. The ongoing valuation in financial markets reflects this fear and shows that it is more responsive to the bad news. Moreover, the recent high expansionary global monetary policy seems like favoring the crash of risk premium. Arthus and Virad (2016) found that when interest rates is too low, investors purchase riskier assets without receiving proper risk premium. Such crash could lead us to inefficient investments.

Under these conditions, it is hard for an econometrician to explain what happens exactly by using traditional econometric methods -the Ordinary Least Squares (OLS), the vector error correction model, the autoregressive distributed lag model, the Granger causality test, among others- because they do not account for possible nonlinearity and asymmetry. Qunatile Regression $(\mathrm{QR})$ can be an alternative to address the issues of nonlinearity and asymmetry since it can account for a set of regression curves that differ across various quantiles of the conditional distribution of the dependent variable, and thus robust to outliers. However, it ignores the possibility that the relationship between the variables could be varied at distinct points in their respective distributions (Sim and Zhou 2015).

Sim and Zhou (2015) introduced Quantile-on-Quantile Regression (QQR) which is conducted by regressing one quantile on the another quantile. Even though this technique is a generalization of the standard $\mathrm{QR}$, it retains more flexibility, and thus could deliver more robust and accurate estimates and provides a measure of average dependence as well as of the upper and lower tail dependence.

Given the emergence of China as a potential contributor to global growth in recent decades, it is expected that the investigation of spillovers from a slowdown in China's GDP growth has attracted particular attention. However, 
most of the assessments seem qualitative and/or descriptive, for instance, reports by International Monetary Fund (2015) and IMF Asia and Pacific Department Regional Economic Outlooks (2014). This study investigates the impact of the shocks to China GDP growth and oil on G7 stock market returns ${ }^{1}$. China encountered a period of high volatility in stock market worsened by macroeconomic slowdown and financial trepidation. It has consumed about 50 percent of the world's raw materials so its economic slowdown has had a conspicuous influence on commodity related sectors: the prices of many commodities started to fall considerably since her economic slowdown.

Most of empirical research supports that the macro-economy is the key of companies' earnings which are a substantial component in equity pricing. Thus, one can expect that changes in oil price which affect the macroeconomy would also result in changes in stock prices. Huang et al. (1996) argue that increase of oil price exerts a negative impact on real output, and then on stock prices due to high operating costs and low earnings. It is supported by Jones and Kaul (1996) which reveals a negative effect of oil shocks on stock market returns in Canada and the United States. Nevertheless, the negative link between oil price and stock returns does not always hold. Some analyses show a positive link between oil price fluctuations and equity markets (Narayan and Narayan 2010, Ono 2011), while the other studies suggest that oil price ambiguously affects stock market returns (Maghyereh 2004, Park and Ratti 2008, Apergis and Miller 2009, Al Janabi et al. 2010, Bouoiyour and Selmi 2016a). Using wavelet approach, Jammazi and Aloui (2010) find a time-variant relationship between oil price and real stock returns in France, Japan, and the United kingdom; a negative relationship is likely to be more pronounced over the pre-1999 period. More recently, Jammazi and Nguyen (2015) performed an improved regime-switching model to explore the dynamic interactions between oil price and stock markets in a sample of oil dependent countries. They showed that equity returns strongly exhibit a regime-switching behaviour, and respond varyingly to oil price changes. They also claimed that the impact of rising oil price on the conditional volatility

\footnotetext{
${ }^{1}$ We focus on G7 countries as increasing energy efficiency has been one of the main goals targeted by their policy makers. These countries could be also largely affected by the China's slowdown to the great trade relationship with China regarding demand and supply. Although these economies have close levels of economic development, different energy and financial policy alternatives may be available (see Lee and Zheng 2011).
} 
of equities is less intense during the bear market phases than the bull market phases.

Such mixed findings derived from standard econometric tools make its assessment difficult. We deal with this difficulty by using QQR which can shed light on the question whether the same quantile of stock returns exhibits distinct response to different quantiles of oil price. OLS and QR are also conducted to compare the results.

Our findings indicate that the Chinese economy and oil price have the negative effect on the G7 stock markets in various ways. In particular, Germany, Italy and Canada are the most sensitive to the slump in oil prices and the China economic downturn. Other countries exhibit a relatively weak response. The remainder of the paper is organized as follows. Section II outlines the methodology and provides a brief data overview. Section III reports the empirical results and Section IV concludes.

\section{Data and Methodology}

Unlike $\mathrm{QR}$, which regresses the $\theta$-quantile of the stock returns on oil price return, QQR regresses the $\theta$-quantile of stock returns on the $\tau$-quantile of oil price returns. Its parameters are indexed by $\theta$ and $\tau$.

Let $S T R$ be the G7 stock returns. We first postulate a model for the $\theta$-quantile of $S T R$ as a function of its history and oil price returns $(\mathrm{Oil})$ denoted as:

$$
S T R_{t}=\beta^{\theta} O i l_{t}+\alpha^{\theta} S T R_{t-1}+\varepsilon_{t}^{\theta}
$$

where $\varepsilon_{t}^{\theta}$ is an error term that has a zero $\theta$-quantile. The history of $S T R$ is used to reflect the potential exogenous variables. The relationship function $\beta^{\theta}(\cdot)$ is unknown since we don't have prior insights on how STR and Oil are interlinked. We linearize the function $\beta^{\theta}(\cdot)$ by taking a first order Taylor expansion around $\tau$-quantile of oil price returns $\left(\mathrm{Oil}^{\tau}\right)$ to analyze the dependence between $\theta$-quantile of the stock returns and the $O i l^{\tau}$ : 


$$
\beta^{\theta}\left(O i l_{t}\right) \approx \beta^{\theta}\left(O i l^{\tau}\right)+\beta^{\theta^{\prime}}\left(O i l^{\tau}\right)\left(O i l_{t}-O i l^{\tau}\right)
$$

We can redefine $\beta^{\theta}\left(O i l^{\tau}\right)$ and $\beta^{\theta^{\prime}}\left(O i l^{\tau}\right)$ as $\beta_{0}(\theta, \tau)$ and $\beta_{l}(\theta, \tau)$ respectively. Then, the Equation (2) can be rewritten as:

$$
\beta^{\theta}\left(O i l_{t}\right) \approx \beta^{\theta}(\theta, \tau)+\beta_{1}(\theta, \tau)\left(O i l_{t}-O i l^{\tau}\right)
$$

Ultimately, substitute Equation (3) into Equation (1) to obtain:

$$
\operatorname{STR}_{t}=\beta^{\theta}(\theta, \tau)+\beta_{1}(\theta, \tau)\left(O i l_{t}-O i l^{\tau}\right)+\alpha(\theta) S T R_{t-1}+\varepsilon_{t}^{\theta}
$$

To determine the risk of Chinese economic slowdown, we adjust the Equation (4) by including an interaction variable (IT). This allows to capture the change in the effect of oil returns on stock returns as a consequence of China shock. A dummy variable (Dummy ${ }_{2011}$ ), which is 1 if $t$ is greater or equal to 2011 and 0 otherwise, is created and applied to the interaction variable to obtain :

$$
S T R_{t}=\beta_{I T}^{\theta}(\theta, \tau)+\beta_{I T}(\theta, \tau)\left(I T_{t}-I T^{\tau}\right)+\alpha_{I T}(\theta) S T R_{t-1}+\varepsilon_{t, I T}^{\theta}
$$

where $I T_{t}$ is equal to $\mathrm{Dummy}_{2011} \mathrm{Oil}_{t}$ and $I T^{\tau}$ is equal to Dummy ${ }_{2011} O i l^{\tau}$

China economic growth is slowing noticeably since 2011. This growth downturn is driven substantially by China's gradual rebalancing from exports to domestic demand, from manufacturing to services, and from investment to consumption. These developments with market concerns about the future performance of the Chinese economy, are prompting spillovers to other economies through trade links, weaker commodity prices, and financial linkages (see Figure 1). 
Figure1. China real GDP growth and rebalancing

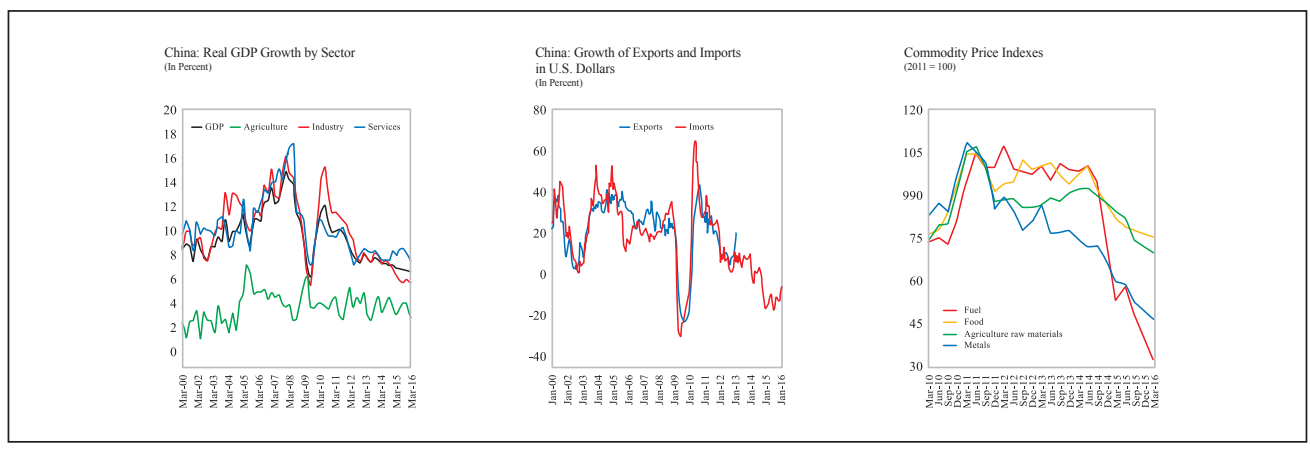

(Source) Dizioli et al. (2016).

Data over the period of January 1999 December $2015^{2}$ for stock prices of France (CAC 40), Germany (DAX 30), Japan (Nikkei 225), the UK (FTSE 100), the United States (Dow Jones), Italy (MIB 30) and Canada (SP-TSX) are employed. The stock market price data are collected from Datastream. For crude oil price return we use the real national price for each country. It has been argued that weekly data is better than daily data for capturing the dynamic interaction among financial and macroeconomic variables, especially commodity prices, as the latter introduces potential biases stemmed from bid-ask effects, nonsynchronous trading days, and the effects of illiquidity on asset prices. Also, weekly data are not subject to volatility transmission mechanisms due to time aggregation and compensation effects (Sadorsky 2014, Antonakakis and Kizys 2015, Batten et al. 2015). Finally, weekly data have been identified as being helpful in capturing the direction of temporal relationships following increased volatility and the transmission of shocks to other markets (Garderbroek et al. 2014).

${ }^{2}$ The period of the study is motivated by the availability of the Italian data and the fact that we required a common sample period for all the G7 countries. 


\section{III.Empirical Results}

\section{A. Benchmark}

This study starts investigation by the use of standard techniques, that is, OLS and QR, with and without IT. The results of QR and OLS without IT are reported in Table 1. Under the OLS estimation, the effect of oil price return on STR seems insignificant in the majority of G7 countries except Japan and Germany. However, it should be noted that OLS estimator focuses only on the central tendency of distribution. OLS does not allow us to examine the dependence between oil price return and G7 equities properly when they are located at non-central regions of its distribution.

Conditional quantile estimates for the focal relationship are also explored by performing QR.The result shows heterogeneous outcomes. In particular, one can distinguish the countries into four groups. The first one includes the United States, France and Italy where one can find a negative dependence between oil price and stock returns at low quantiles (0.1, 0.2 and 0.3, i.e., when the stock market perform worse). The second group is formed by Canada and United Kingdom where a negative connection is found at low quantiles while a positive connection is shown at medium and upper quantiles (when the stock market perform normally or are in a better performance). The third group contains Germany in which oil price return is negatively correlated to stock return at upper quantiles of STR. The fourth group includes Japan where a strongly significant positive linkage between oil price return and equity is revealed at all quantiles.

When the recent Chinese economic downturn is incorporated, the relationship between $\mathrm{Oil}$ and $S T R$ increases sharply for all the countries as shown in Table 2. Unlike the previous results, it is difficult to classify countries into coherent groups but one can notify that the impact of Oil on the United States STR is negative under bear stock market and positive when the stock market is improving. For the United Kingdom, oil price shocks exert a negative impact on stock returns when the stock market is at bearish or normal phase. Oil adversely affects German STR when the stock market is declining, whereas the impact of Oil on Franch stoch markets is positive under bear and normal market phases. For Italy, Oil negatively affects STR when the stock market is bullish. 
In short, Table 2 shows how and to what extent the combined effect of the Chinese economic slowdown and oil price changes affects the G7 stock returns. Whatever quantiles one chooses, shocks to China GDP growth and oil affects markets significantly and negatively all the G7 stock. Germany suffered the most from Chinese economic downturn. It should be also pointed out that OLS estimates are statistically significant for only three countries (the USA, the UK and France). 


\begin{tabular}{|c|c|c|c|c|c|c|c|c|c|c|}
\hline \multirow{7}{*}{ 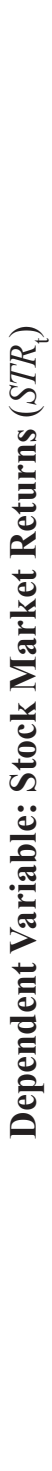 } & 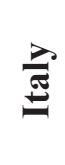 & 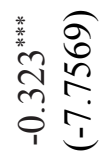 & 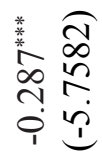 & 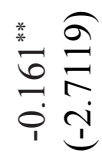 & 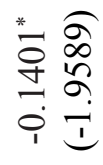 & 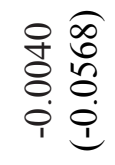 & 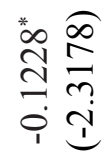 & 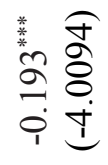 & 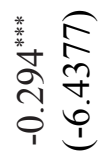 & 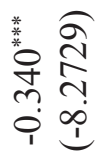 \\
\hline & 导 & 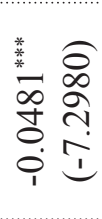 & 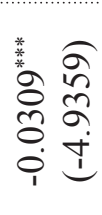 & 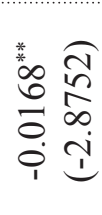 & 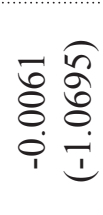 & 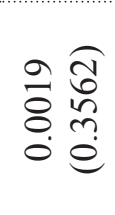 & 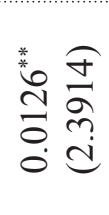 & 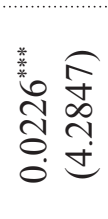 & 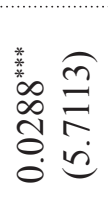 & \begin{tabular}{l}
$\hat{\sigma}$ \\
\multirow{0}{*}{} \\
0 \\
0 \\
0
\end{tabular} \\
\hline & 总 & 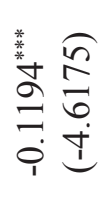 & 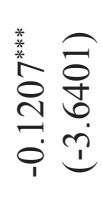 & 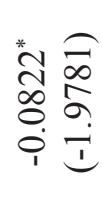 & 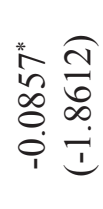 & 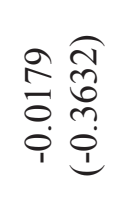 & 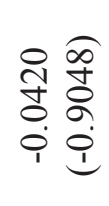 & 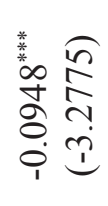 & 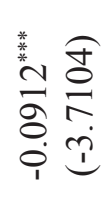 & 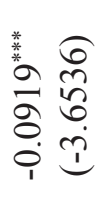 \\
\hline & 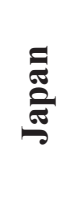 & 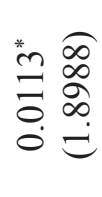 & 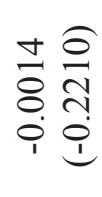 & 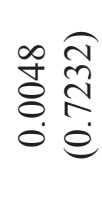 & \begin{tabular}{l}
\multirow{2}{*}{} \\
$\stackrel{n}{0}$ \\
0 \\
0 \\
0
\end{tabular} & 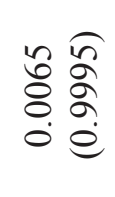 & 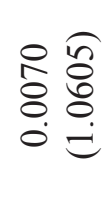 & 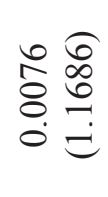 & 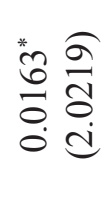 & 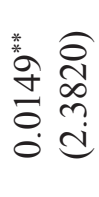 \\
\hline & ָֻ & $\begin{array}{l}\hat{0} \\
\hat{0} \\
0 \\
0 \\
0 \\
0\end{array}$ & 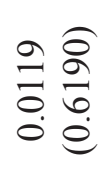 & 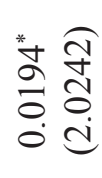 & 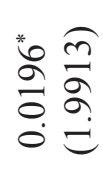 & 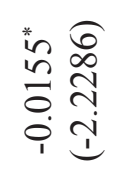 & 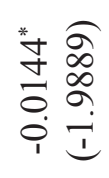 & 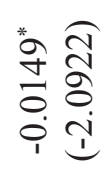 & 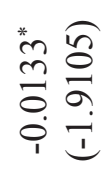 & 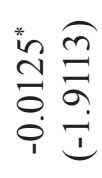 \\
\hline & 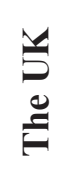 & 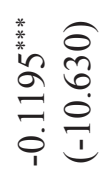 & 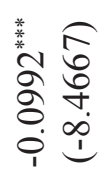 & 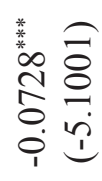 & 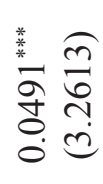 & 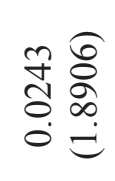 & 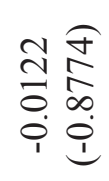 & 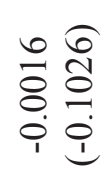 & 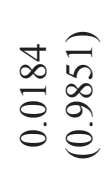 & 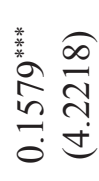 \\
\hline & 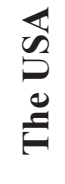 & 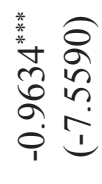 & 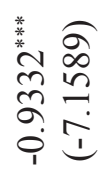 & 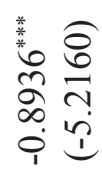 & 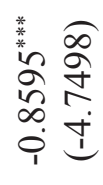 & 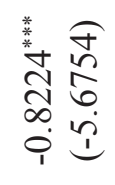 & 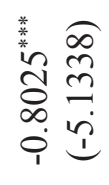 & 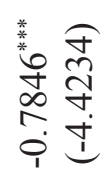 & 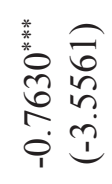 & 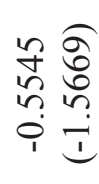 \\
\hline \multicolumn{2}{|c|}{ 总 } & $\overrightarrow{0}$ & $\stackrel{\Upsilon}{0}$ & $\stackrel{n}{0}$ & $\stackrel{\nabla}{\circ}$ & $\stackrel{n}{0}$ & $\stackrel{0}{0}$ & $\dot{\sigma}$ & $\stackrel{\infty}{\circ}$ & $\stackrel{\varrho}{0}$ \\
\hline \multicolumn{2}{|c|}{$\frac{0}{\frac{0}{0}}$} & \multicolumn{9}{|c|}{ 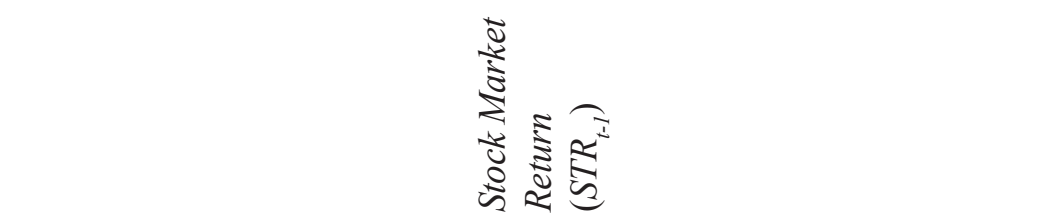 } \\
\hline
\end{tabular}




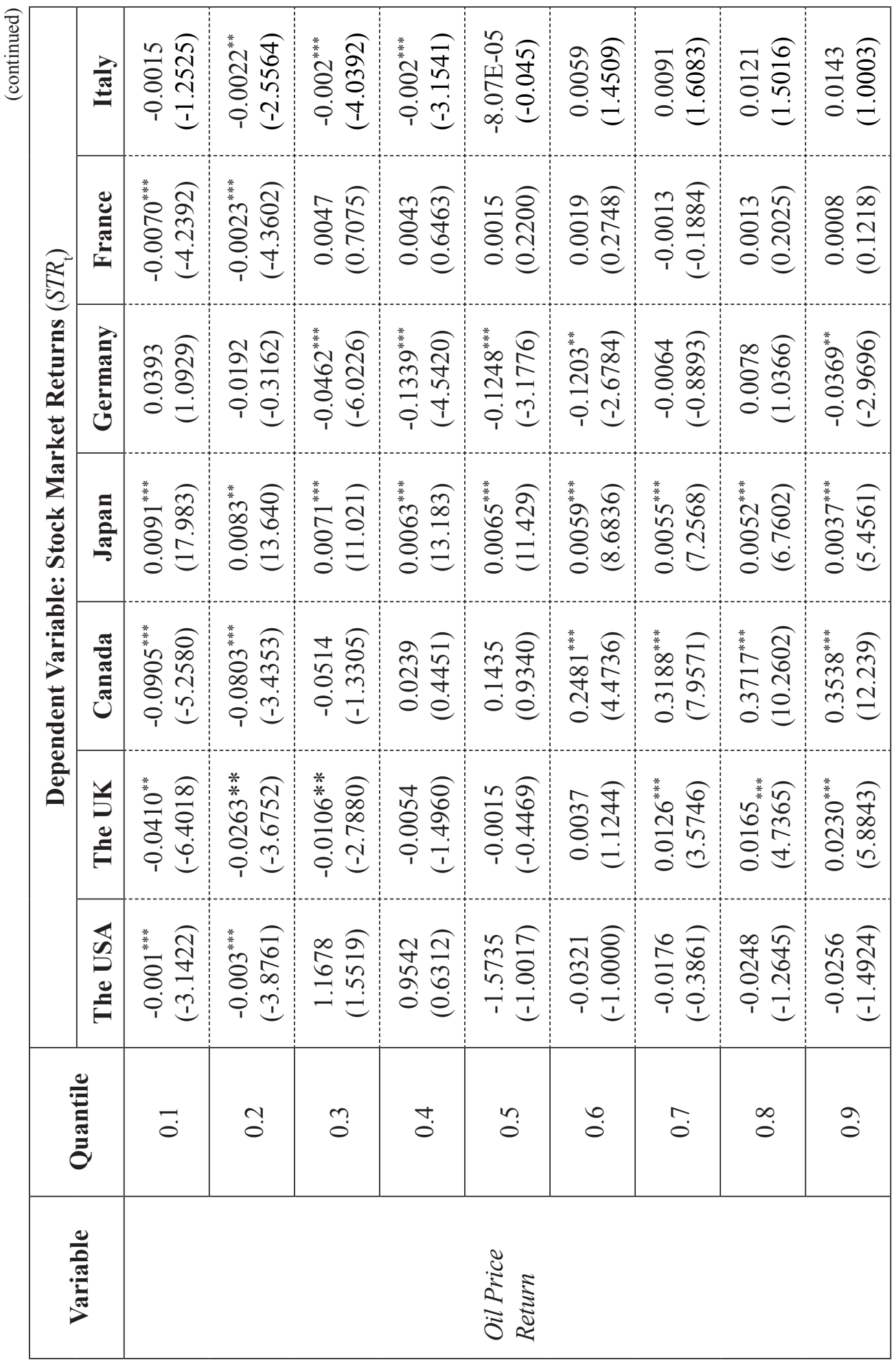




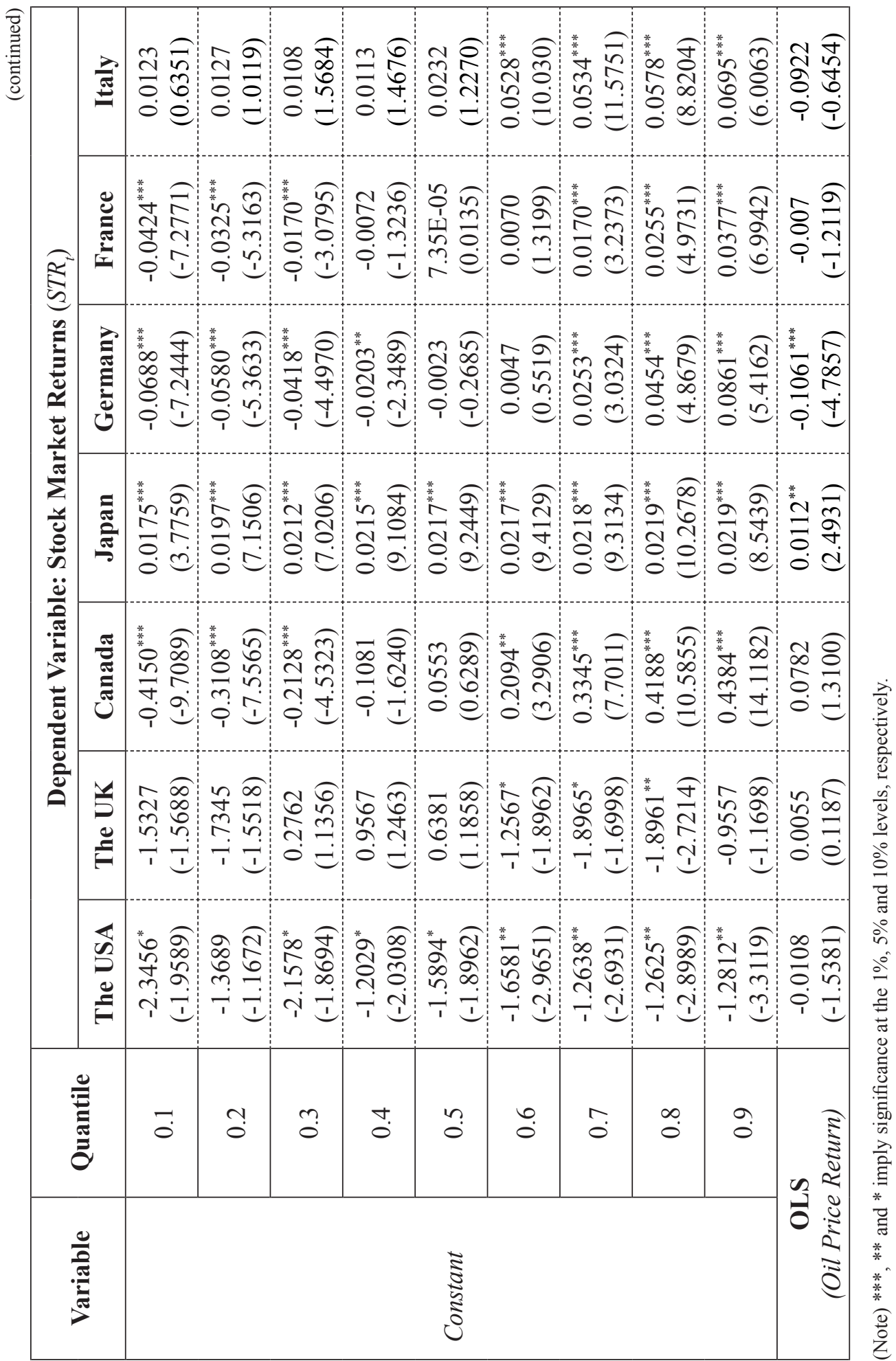




\begin{tabular}{|c|c|c|c|c|c|c|c|c|c|c|}
\hline & $\stackrel{\frac{\lambda}{\sigma}}{\underline{\sigma}}$ & 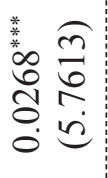 & 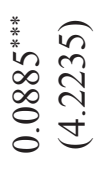 & 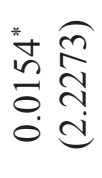 & 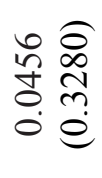 & 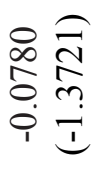 & 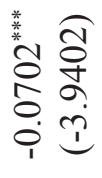 & 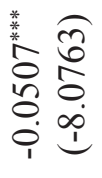 & 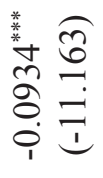 & 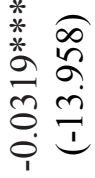 \\
\hline$\overparen{\nabla}$ & 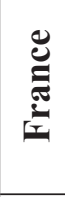 & 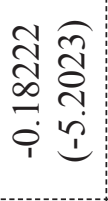 & 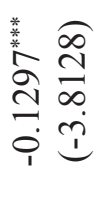 & 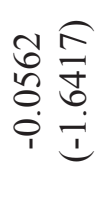 & 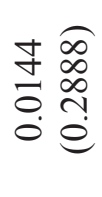 & 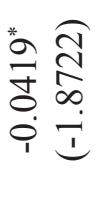 & 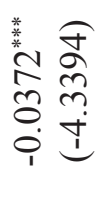 & 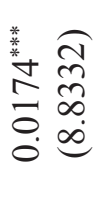 & 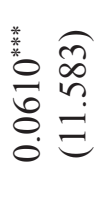 & 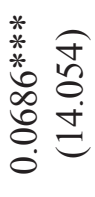 \\
\hline 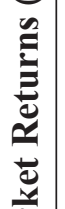 & 预 & 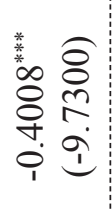 & 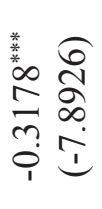 & 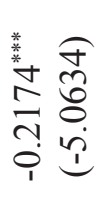 & 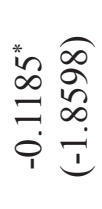 & $\begin{array}{ll}m & 0 \\
n & 6 \\
0 & n \\
0 & 0\end{array}$ & 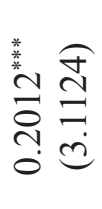 & 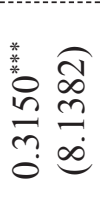 & 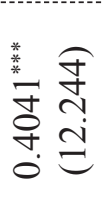 & 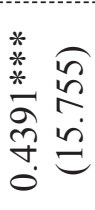 \\
\hline $\begin{array}{l}\bar{\Xi} \\
\sum \\
\bar{e} \\
\dot{0} \\
\ddot{0} \\
\ddot{0}\end{array}$ & 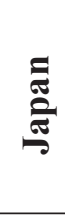 & 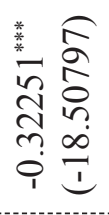 & 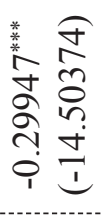 & 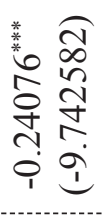 & 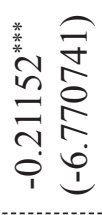 & 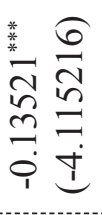 & 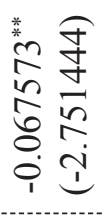 & 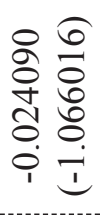 & 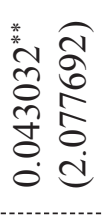 & 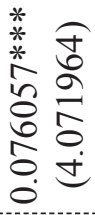 \\
\hline 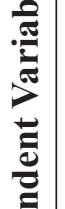 & Uુ & 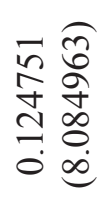 & 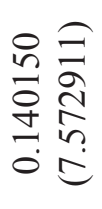 & 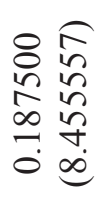 & 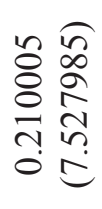 & 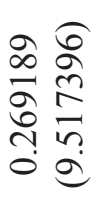 & 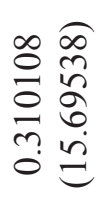 & 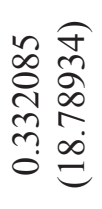 & 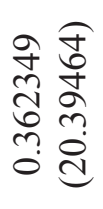 & 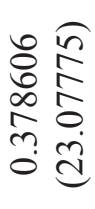 \\
\hline$\frac{0}{0}$ & 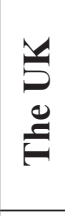 & 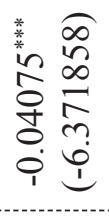 & 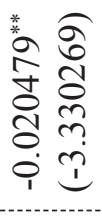 & 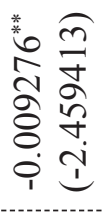 & 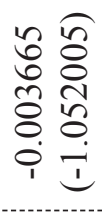 & 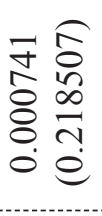 & 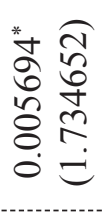 & 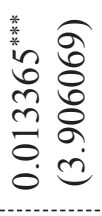 & 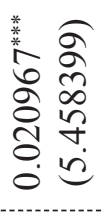 & 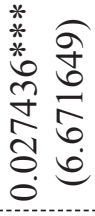 \\
\hline & 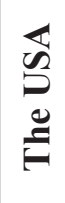 & 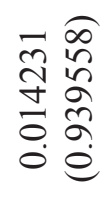 & 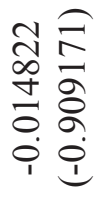 & 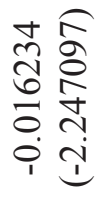 & 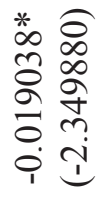 & 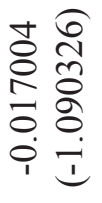 & 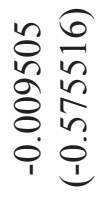 & 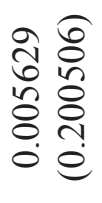 & 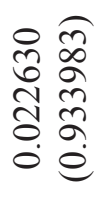 & 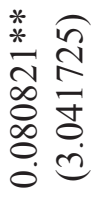 \\
\hline & שֶ. & $\overrightarrow{0}$ & ֻ̦ & $\stackrel{n}{0}$ & $\stackrel{+}{\circ}$ & $\stackrel{n}{0}$ & $\stackrel{0}{0}$ & $\dot{0}$ & $\stackrel{\infty}{0}$ & $\stackrel{\varrho}{0}$ \\
\hline & $\underline{\underline{\sigma}}$ & \multicolumn{9}{|c|}{ 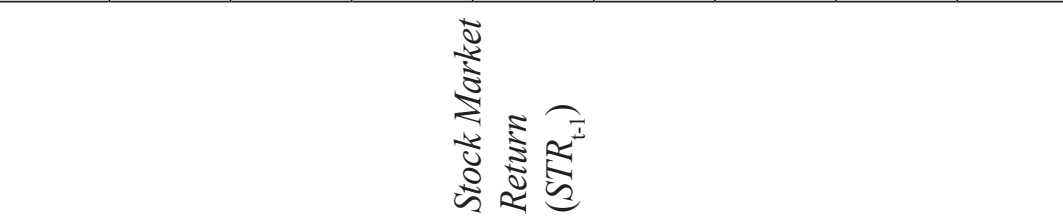 } \\
\hline
\end{tabular}




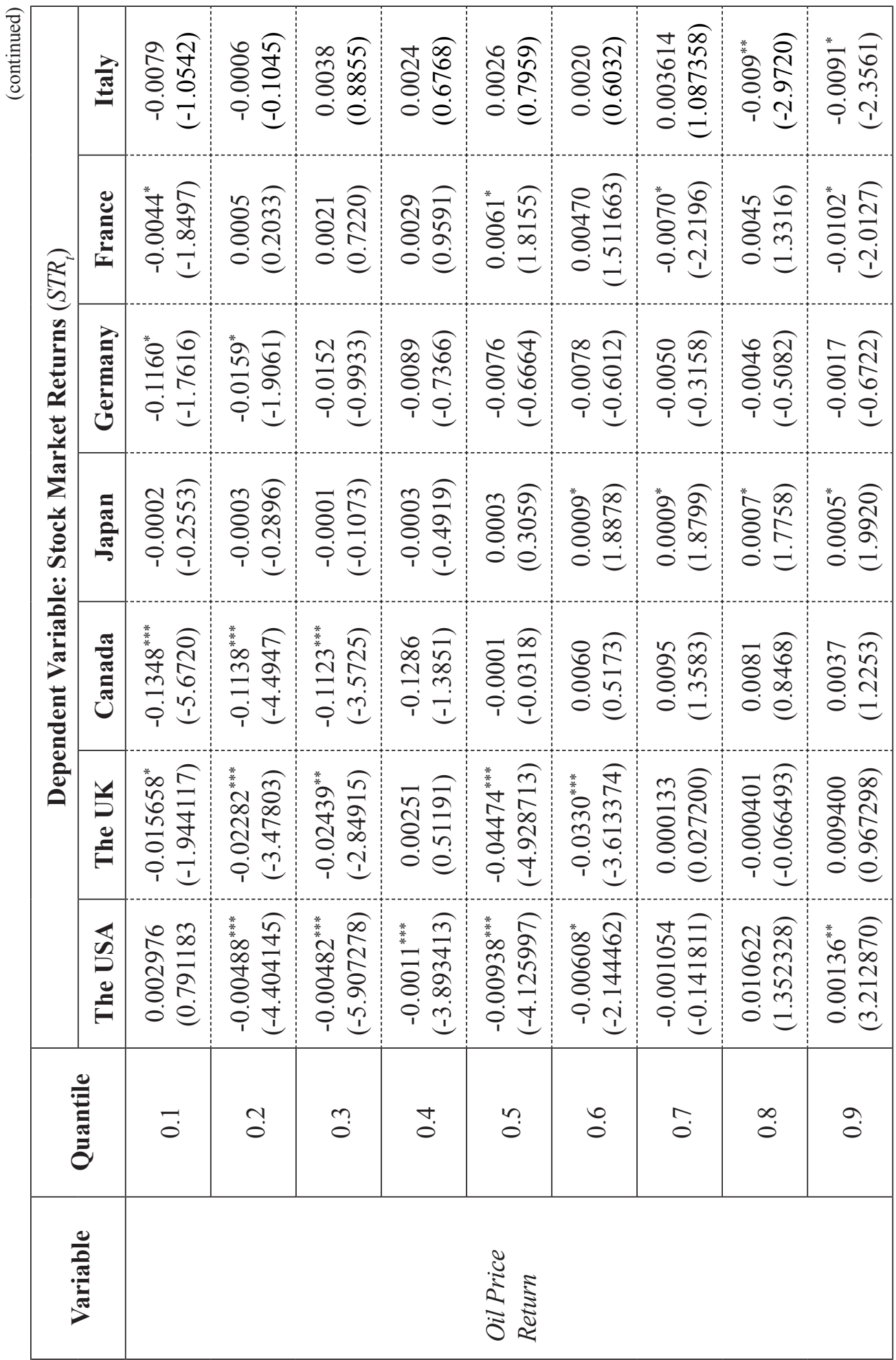




\begin{tabular}{|c|c|c|c|c|c|c|c|c|c|}
\hline 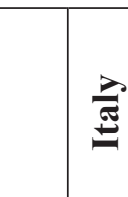 & 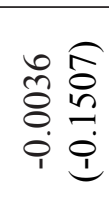 & 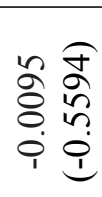 & 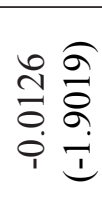 & 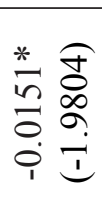 & 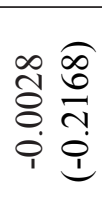 & 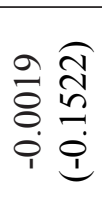 & 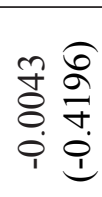 & 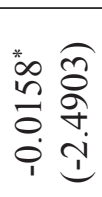 & 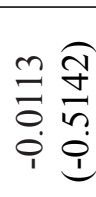 \\
\hline \multirow{6}{*}{ 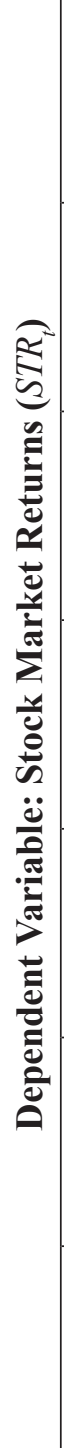 } & 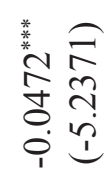 & 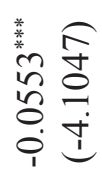 & 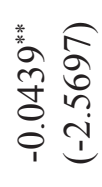 & 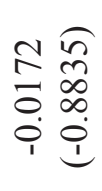 & 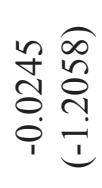 & 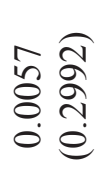 & 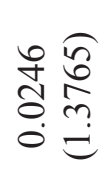 & 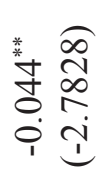 & $\begin{array}{l}\hat{n} \\
\text { กิ } \\
0 \\
0 \\
0\end{array}$ \\
\hline & 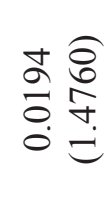 & 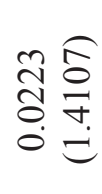 & 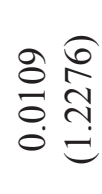 & 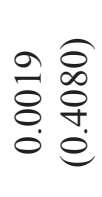 & 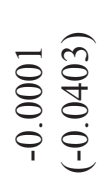 & 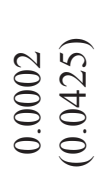 & 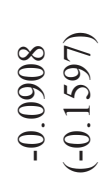 & 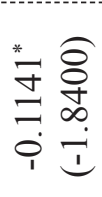 & 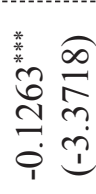 \\
\hline & 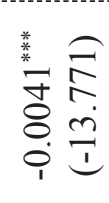 & 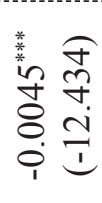 & 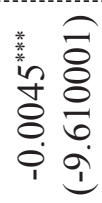 & 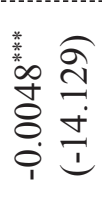 & 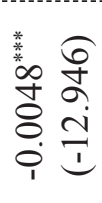 & 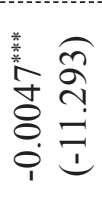 & 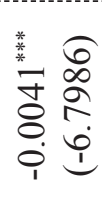 & 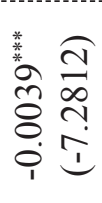 & 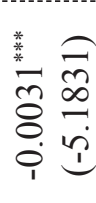 \\
\hline & 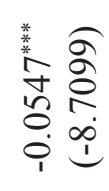 & 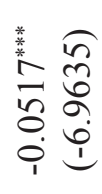 & 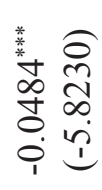 & 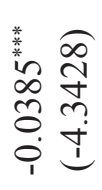 & 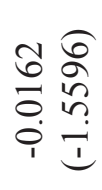 & 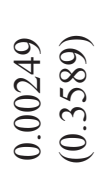 & 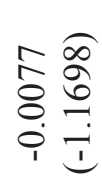 & 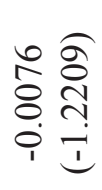 & 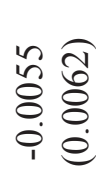 \\
\hline & 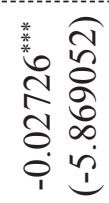 & 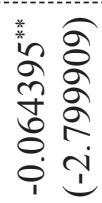 & 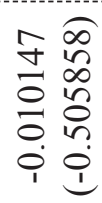 & 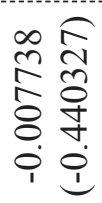 & $\begin{array}{ll}\bar{a} & \hat{\circ} \\
\hat{\sigma} & \hat{o} \\
8 & 0 \\
0 & 0 \\
0 & \dot{e}\end{array}$ & 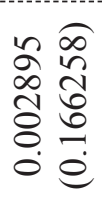 & 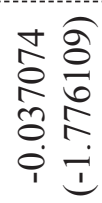 & 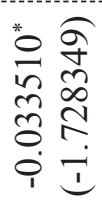 & 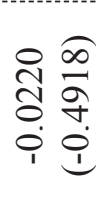 \\
\hline & 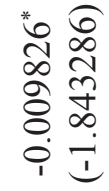 & 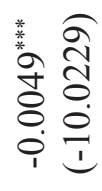 & 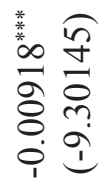 & 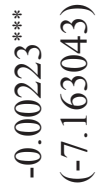 & 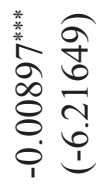 & 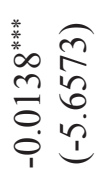 & 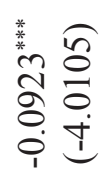 & 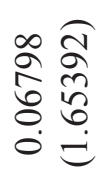 & 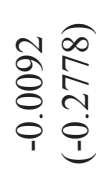 \\
\hline 鸹 & $\overrightarrow{0}$ & $\stackrel{\Upsilon}{0}$ & $\stackrel{n}{0}$ & $\stackrel{\nabla}{\circ}$ & n? & $\stackrel{0}{0}$ & $\dot{0}$ & $\stackrel{\infty}{\circ}$ & $\stackrel{\varrho}{0}$ \\
\hline 党 & & & & & 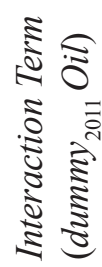 & & & & \\
\hline
\end{tabular}




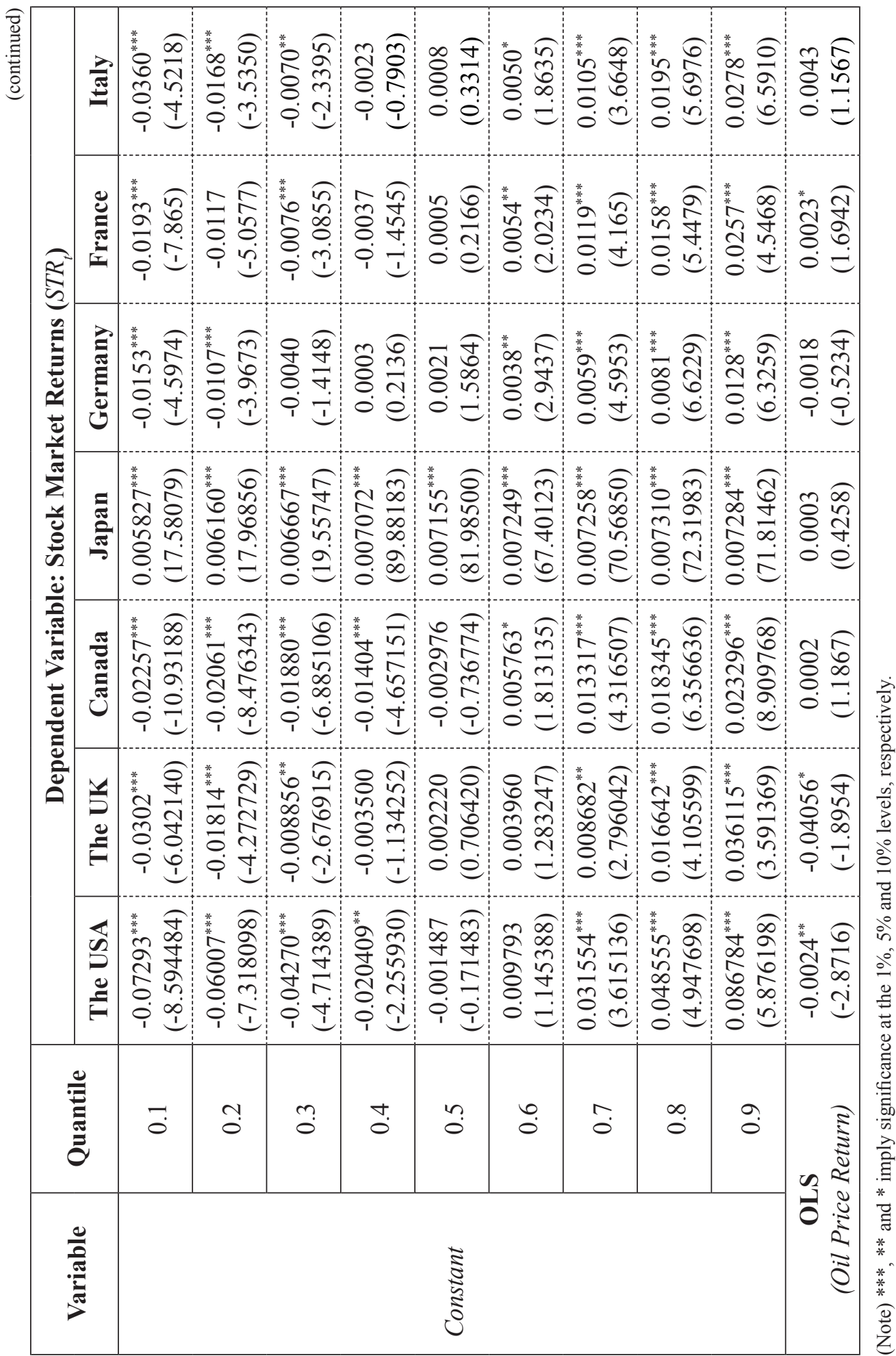




\section{B. $Q Q R$ results}

Oil price fluctuations do not uniformly affect the $\mathrm{G} 7$ equities as shown in Figure 2. In particular, the countries can be categorized into two groups. The first includes Germany, Italy, Canada and United Kingdom in this order, where the effect of oil price return appears strong. The second group is formed by France, Japan, and the United States; the range of the coefficient of $S T R$ is from -0.10 to 0.20 in France; from -0.10 to 0.12 in Japan; from -0.05 to 0.07 in the United States.

One can expect that low oil price have a positive effect on stock returns of the US, Germany, Japan, France and Italy since they are oil importing countries and have a negative effect on that of Canada and the UK because they are oil exporting countries. However, our findings exhibit different results and highlight the complexity of the focal relationship. For the USA, when oil price return is in the medium or high level (quantiles of 0.5, 0.6 and $0.7)$ the stock returns react positively while when oil price return is in the low quantiles $(0.1$ and 0.2$)$ the reaction appears negative or negligible. When oil price is at the upper quantile ( 0.8 and 0.9 ) the response of stock return is very modest or negligible. Similar results are found in Japan. If we focus on the United Kingdom, oil returns exert a negative impact on STR when the market is improving (quantile of 0.9). Likewise, for Germany and Italy, a negative response is found under bull market conditions.

For Canada, the reaction of stock returns to oil price return is often positive either when investors are optimistic (STR is in high quantiles) or pessimistic (STR is in low quantiles), which is theoretically unanticipated. However, the correlation is relatively modest or negative when the stock market is normal or mildly bullish (for STR quantiles 0.5, 0.6). For France, when investors are pessimistic (for quantiles 0.3 and 0.4 ), the stock response to oil price changes seems negative, while it appears positive when investors are optimistic (for STR quantiles 0.8 and 0.9). Nevertheless, the response of Italian stock returns to oil price returns is negative whatever the stock market circumstances, except when the oil price is high (quantiles 0.8 and 0.9).

Overall, our results show that the dependence structure between stock returns and oil shocks in uncertain episodes, in particular, in a period of a slowing chinese economy, depends on the varying stock market states, and the nuances of oil prices. In addition the relationship is nonlinear, probably 
due to the mood of stock investors, the emergence of gas shales that profoundly transforms the industries, the interplay of supply and demand in the oil market, the price fluctuations of other assets and the adoption of unconventional monetary policies.

\section{Figure 2. Quantile-on-Quantile regression without interactive term}

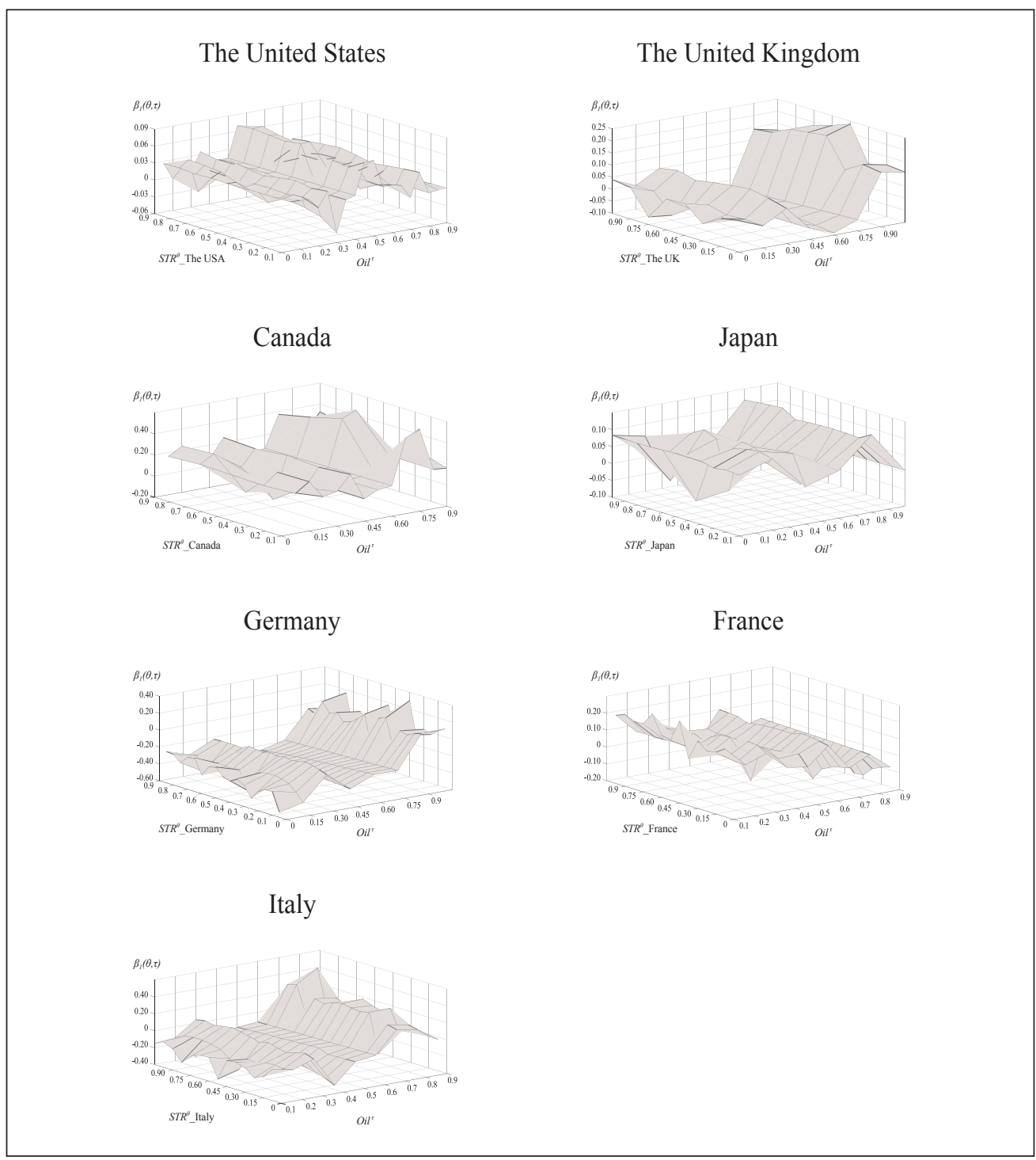


Figure 3 shows the results when Chinese economic slowdown is incorporated. The dependence between oil price return and stock return increases markedly for the countries under investigation. Italy where the strength of the relationship fell and France where the linkage appears unvarying are only exceptions. We are able to keep the same group categoty as in the analysis without $I T$ with slight changes. More accurately, the response of STR to Oil is stronger for the group of countries formed by Germany, Canada, the United Kingdom and Italy in this order. Nevertheless, the focal relationship appears weaker for the group constituted by Japan, the USA and France. The interaction effect of China's economics slowdown and oil price return does not change our results substantially. We also find that Germany is likely to be more jeopardized than Italy and Canada; the responses of STR to Oil rangefrom -0.60 to 0.40 for Italy; from -0.50 to 0.50 for Canada. These three are the most affected countries by China and oil shocks.

The aforementioned findings support the relevance of QQR to uncover the characteristics of the dependence between G7 stock returns and oil price returns that cannot be identified by OLS or QR. For example, the OLS results indicate that the effect of oil price return on G7 stock returns is insignificant or at most moderate. QR results suggest that oil price return has variant effect on $\mathrm{G} 7$ stock returns depending to the states of G7 stock markets. We go beyond, by applying QQR, Oil-STR relationship is conditional on the different G7 equities scenarios (bear, normal or bull regimes), the various kinds of oil price level (low, normal or high) and the heightened uncertainty surrounding Chinese economic slowdown.

Figure 3. Quantile-on-Quantile regression without interaction term

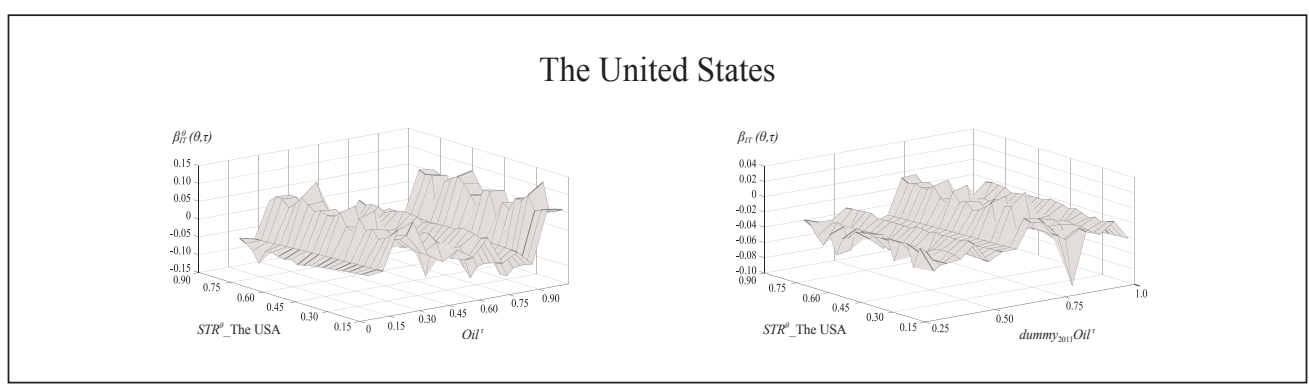




\section{United Kingdom}
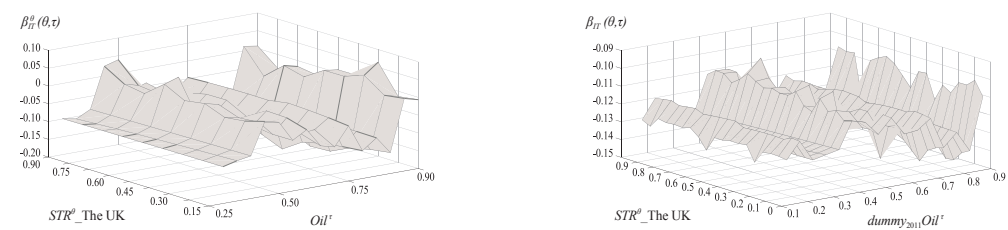

Canada
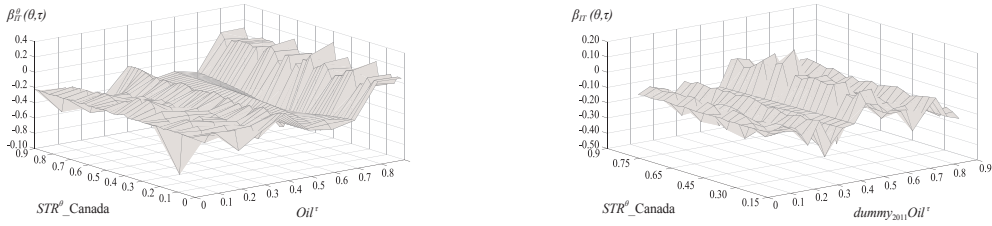

Japan
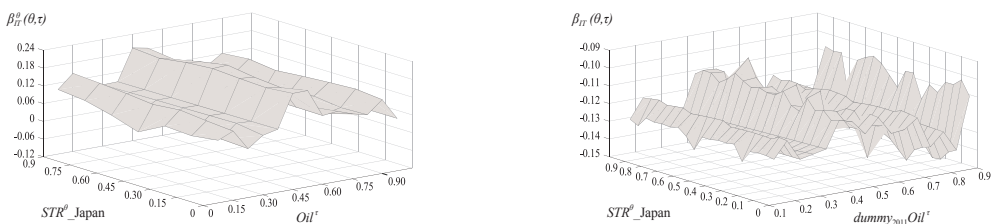

Germany
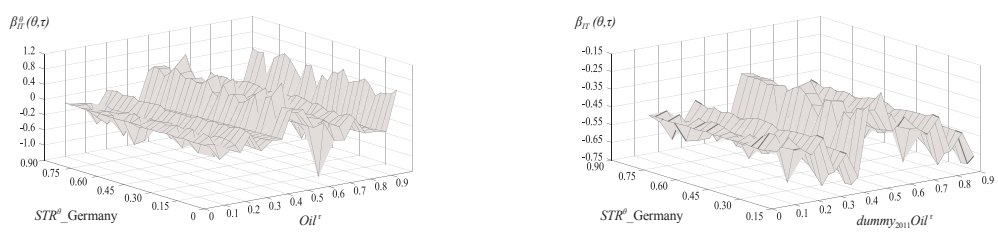

\section{France}
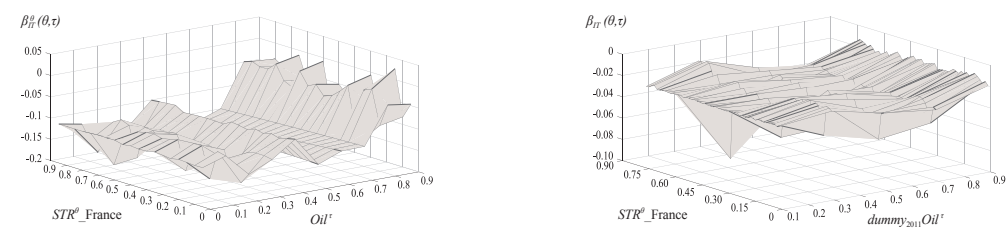

Italy
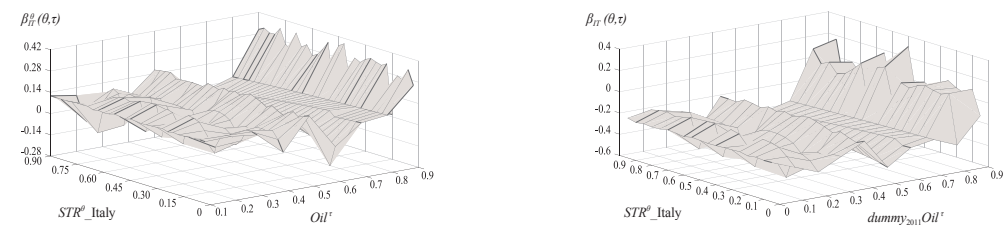


\section{Discussion}

The different responses of G7 stock returns to oil price return may be attributed to the current account position of the country and the extent of oil dependency, which were largely offered in previous studies (for example, Park and Ratti 2008, Kilian and Park 2009, Nandha and Brooks 2009, Jammazi and Nguyen 2015). Additional channels through which oil price return may varyingly affect stock returns is advanced by Bouoiyour and Selmi (2016b). These channels include the concentration level on oil-based revenues and the skewed distribution of cyclical and noncyclical sectors.

Our results support that the G7 stock returns respond significantly to the Chinese economic slowdown. Consistent with O'Neil et al. (2015), we show that this link is sensitive to whether market expectations are overly optimistic (bull) or pessimistic (bear) with regards to the future Chinese GDP growth and to the nuances of oil price.

Various channels can be identified (Heyer 2015) to explain. Given the leading role of China in the world economy, the fact that China's economy shows signs of slowing down since the first quarter of 2011 would hinder its imports and deteriorate the current account position of exporters. This might also affects the Foreign Direct Investments, which would increase capital outflows, prompt a new international distribution of assets, and exacerbate the contagion effect across financial markets. Further, since almost the half of all metals traded in the world is purchased by China, its recent economic upheaval and the downward adjustments in its industry may cut demand for some commodities, especially oil and petroleum products.

We expected that Chinese economic downturn would affect Germany the most (35.9 billion euros or 45 percent of European export goods to China in 2014) followed by the United Kingdom (19.6 billion euros or 12.3 percent), France (16.2 billion euros or 10.6 percent) and then Italy ( 10.5 billion euros or 6.8 percent) (Eurostat 2015). However, results do not validate this hierarchy:German, Italy and Canada suffered the most from the Chinese collapse.

The fact Germany is more damaged is not surprising considering its position as European largest exporter. It is also the biggest importer of Chinese goods absorbing almost 20 percent of the European imports from China, and has also been one of the most active European countries in 
investing in China. The fact that France appears less influenced may reflect that France assumed the leading role only recently (since 2015).

Italy is the Chinese fourth largest trading partner in the European Union and share a history of friendly exchanges with it. Although the development of China-Italy relation has been relatively steady, these two economies are facing various problems that may exacerbate the impacts of the recent Chinese upheaval on Italian market. Italy has lacked the capacity to exploit the opportunities offered by Chinese economy. Specifically, the Italian entrepreneurial and institutional systems appear less than dynamic with respect to the agility to these opportunities. Despite the encouraging prospects arising from new sectors of Chinese-Italian economic cooperation, Italy's lack of distribution channels, underdeveloped market and the unsystematic operation of her corporate sector over a tightly-knit institutional setup have often damaged the relationship (Andornino 2012).

Canada is the third country which appears heavily affected by China's slowdown. Given the economic complementarities between both countries, this result seems obvious. The energy sector is the deepest game changer for their bilateral relationship. China is turning to clean energy sources that Canada could provide innovative ideas (Dobson and Evans 2015).

For the United Kingdom, despite the harmful consequences of the European economic crisis, it has not been receiving investments from China by seeking other methods to eliminate the mounting trade deficit known as "Brexit".

Last but not least, being less affected by Chinese economic downturn implies numerous sector-specific restrictions of market access taken by China (Barone and Bendini 2015).

\section{Conclusion}

We show that the responses of G7 equities to China and oil shocks is asymmetric, nonlinear and country-specific. The stock market returns of Germany, Italy and Canada respond more adversely to the slump in oil prices and the China's economy slow-moving. Other countries show a moderate response. The strength and the depth of the China-G7 economic relationships and the oil reliance have been addressed to explain the heterogeneous 
reactions of G7 stock markets. For example, in countries highly dependent on commodity exports (like Canada), the slowdown could have been more pronounced.

International investors can brace against some of the implication of a slowdown in the Chinese economy by taking appropriate measures aimed to rebalancing their portfolio. This article provides information about when the relation between G7 stock return and China and oil shocks is negative, when it is positive, when it is modest, and when it is strong, depending on the quantiles of oil price returns and G7 stock returns. This would allow to clarify stock market structure on the multi-dimensional basis. The evidence that the G7 equities react heterogeneously to oil price and Chinese economic downturn would help to construct an optimal portfolio. The international investors should be cautious about the significant dependence between stock returns and Chinese slowdown, especially when they invest in Germany, Canada and Italy whose stock markets suffered most from the Chinese economic slowdown. Also, the different influences of the oil price measures (in US dollar, pound sterling, Canadian dollar, yen, euro) on stock returns suggest a potential substitution among these oil markets, and thus, it may be opportune for energy policymakers to diversify the origins of oil imports to avoid unforeseen oil shocks. Regulators could build a long-run international investors' confidence in the stock markets safeguarding against possible risk.

Although our analysis focuses only on seven largest economies in the world, which take a share of more than $46 \%$ of the global GDP, our findings can be extended to other emerging and developing countries. During the last years, China has integrated strongly not only with the G7 countries but also with the rest of the world. Other regions such as Asia, Africa and the Middle East, also have a reason to concern about the possible detrimental effects of the slowdown in the world's second largest economy. This could be identified as an area for future research.

Received 25 January 2018, Revised 5 July 2018, Accepted 29 August 2018 


\section{References}

Al Janabi, M.A.M., Hatemi, J.A., Irandoust, M., "An empirical investigation of the informational efficiency of the GCC equity markets: evidence from bootstrap simulation." International Review of Financial Analysis 19 (2010): 47-54.

Apergis, N., Miller, S.M., "Do structural oil-market shocks affect stock prices?" Energy Economics 31 (2009): 569-575.

Andornino, G., "The political economy of Italy's relations with China: the International Spectator." Italian Journal of International Affairs 47 (2) (2012) : 87-101.

Antonakakis, N., and Kizys, R., "Dynamic spillovers between commodity and currency markets." International Review of Financial Analysis 41 (2015) : 303319.

Arthus, P. and Virad M-P., "La folies des banques centrales."Fayard Edition (2016).

Basistha, A. and Kurov, A., "Macroeconomic Cycles and the Stock Market Reaction to Monetary Policy." Journal of Banking \& Finance(2016).

Barone, B. and Bendini, R. "China: Economic outlook." the European Parliament's Delegation for relations with the People's Republic of China(2015).

Batten, J.A., Ciner, C., and Lucey, B.M., "Which precious metals spill over on which, when and why? Some evidence." Applied Economics Letters (2015) : 466-473.

Bouoiyour J. and Selmi, R., "Testing for Frequency Causality between Oil Price and BRICS Stock Markets: A Comparative Analysis." Journal of Economic 
Integration31 (3) (2016a): 547-568.

Bouoiyour, J. and Selmi, R. "China's slowdown and Uncertainty Spillovers within BRICS stock markets: A Signal processing analysis." Working paper CATT, University of Pau(2016b).

Dizioli, A., J. Guajardo, V. Klyuev, R. Mano, and Raissi, M., "Spillovers from Chinaäs Growth Slowdown and Rebalancing to the ASEAN-5 Economies." IMF Working Paper 16/170 (2016).

Dobson, W., and Evans, P., "L'avenir des relations Canada-Chine." Institut de recherché en politiques publiques, IRPP Working paper. Available at: http:// irpp.org/fr/research-studies/lavenir-des-relations-canada-chine/

Garderbroek, C., Hernandez, M. A., and Robles, M., "Market interdependence and volatility transmission among major crops." Discussion paper $\mathrm{n}^{\circ} 01344$ (2014).

Heyer É., "L'ampleur du ralentissement chinois et son impact sur les grands pays développés, une analyse économétrique.” Revue de l'OFCE 144 (2015).

Huang, R.D., Masulis, R.W., Stoll, H.R., "Energy shocks and financial markets.” Journal of Futures Markets 16 (1996):1-27.

International Monetary Fund, "India Selected Issues: Spillover Analysis." IMF Country Report No. 14/58 (2014).

International Monetary Fund, "China: 2015 Article IV Consultation." IMF Country Report No. 15/234 (2015).

Jammazi, R., and Aloui, C., "Wavelet decomposition and regime shifts: Assessing the effects of crude oil shocks on stock market returns." Energy Policy 38 (2010): 1415-1435. 
Jammazi, R., and Nguyen, D-K., "Responses of international stock markets to oil pricesurges: a regime-switching perspective." Applied Economics (2015); DOI: $0.1080 / 00036846.2015 .1030566$.

Jones, C.M., Kaul, G., "Oil and the stock markets." Journal of Finance 51 (1996): 463-491.

Lee, C. C., Zeng, J. H., "The impact of oil price shocks on stock market activities: Asymmetric effect with quantile regression." Mathematics \& Computers in Simulation 81(2011): 1910-1920.

Maghyereh, A., "Oil Price Shocks and Emerging Stock Markets: A Generalized VAR Approach, International Journal of Applied Econometrics and Quantitative Studies." Euro-American Association of Economic Development 1(2004): 27-40.

Nandha, M., Faff, R., "Does oil move equity prices? A global view.” Energy Economics 30 (2008): 986-997.

Narayan, P.K., Narayan, S., "Modelling the impact of oil prices on Vietnam's stock prices." Applied Energy 87 (2010): 356-361.

Ono, S., "Oil price shocks and stock markets in BRICs." European Journal of Comparative Economics 8 (2011): 29-45.

O’Neill, Stupnytska \& Wrisdale "Linking GDP Growth and Equity Returns." Goldman Sachs Asset Management Monthly Insights (2011).

Park, J., Ratti, R.A., "Oil price shocks and stock marketsin the U.S. and 13 European countries.” Energy Economics 30 (2008) : 2587-2608.

Sadorsky, P., "Modeling volatility and correlations between emerging market stock prices and the prices of copper, oil and wheat." Energy Economics 43 (2014): 72-81. 
Sim, N., and Zhou, A., "Oil prices, US stock return, and the dependence between theirquantiles." Journal of Banking \& Finance 55 (2015) : 1-8. 